\title{
Randomized Controlled Trial to Test the Efficacy of an Unguided Online Intervention with Automated Feedback for the Treatment of Insomnia
}

\author{
Lorenz, Noah ; Heim, Eva ; Roetger, Alexander ; Birrer, Eva ; Maercker, Andreas
}

\begin{abstract}
BACKGROUND: Insomnia has become a major public health concern. AIMS: The study examined the efficacy of a web-based unguided self-help programme with automated feedback. The programme was based on cognitive behaviour therapy for insomnia (CBT-I). The investigation particularly focused on factors that contribute to the maintenance of insomnia and tested whether treatment effects were stable over a period of 12 months. METHOD: Fifty-six participants were randomly assigned either to web-based CBT-I or to the waiting-list control group. Included measures assessed insomnia severity, sleep-related cognitions, safety behaviours, depression, anxiety and somatization. In the intervention group, a sleep diary was used to assess sleep continuity parameters, sleep quality and daytime performance. RESULTS: Large between- and within-group effect sizes $(\mathrm{d}=1.79, \mathrm{~d}=1.59)$ for insomnia severity were found. The treatment group effect remained stable over the period of 12 months. Further, sleep-related cognitions, safety behaviours, depression and somatization significantly decreased in the treatment group compared with the control group. On all sleep diary parameters, medium to large effects were revealed within the treatment group. Anxiety did not decrease significantly from pre- to post-assessment. For all measures except somatization and anxiety significant within-group effects were found at 12-month follow-up assessment indicating long-lasting effects. CONCLUSIONS: This study adds evidence to the literature on unguided online interventions for insomnia, and indicates that online CBT-I can have substantial long-term effects on relevant sleep-related outcome parameters. Moreover, the results indicate that sleep-related cognitions and safety behaviour can be successfully altered with an unguided CBT-I intervention.
\end{abstract}

DOI: https://doi.org/10.1017/S1352465818000486

Posted at the Zurich Open Repository and Archive, University of Zurich

ZORA URL: https://doi.org/10.5167/uzh-157067

Journal Article

Published Version

Originally published at:

Lorenz, Noah; Heim, Eva; Roetger, Alexander; Birrer, Eva; Maercker, Andreas (2019). Randomized Controlled Trial to Test the Efficacy of an Unguided Online Intervention with Automated Feedback for the Treatment of Insomnia. Behavioural and Cognitive Psychotherapy, 47(3):287-302.

DOI: https://doi.org/10.1017/S1352465818000486 


\title{
Randomized Controlled Trial to Test the Efficacy of an Unguided Online Intervention with Automated Feedback for the Treatment of Insomnia
}

\author{
Noah Lorenz \\ Medical Faculty, Department of Psychiatry and Psychotherapy, University Leipzig, Leipzig, Germany
}

\author{
Eva Heim
}

Department of Psychology, University of Zurich, Switzerland

\author{
Alexander Roetger \\ Department of Psychology, University of Zurich, Switzerland
}

Eva Birrer

Seeklinik Brunnen, Gersauerstrasse 8, 6440 Brunnen, Switzerland

Andreas Maercker

Department of Psychology, University of Zurich, Switzerland

\begin{abstract}
Background: Insomnia has become a major public health concern. Aims: The study examined the efficacy of a web-based unguided self-help programme with automated feedback. The programme was based on cognitive behaviour therapy for insomnia (CBT-I). The investigation particularly focused on factors that contribute to the maintenance of insomnia and tested whether treatment effects were stable over a period of 12 months. Method: Fifty-six participants were randomly assigned either to web-based CBT-I or to the waiting-list control group. Included measures assessed insomnia severity, sleep-related cognitions, safety behaviours, depression, anxiety and somatization. In the intervention group, a sleep diary was used to assess sleep continuity parameters, sleep quality and daytime performance. Results: Large between- and within-group effect sizes $(d=1.79, d=1.59)$ for insomnia severity were found. The treatment
\end{abstract}

Correspondence to Noah Lorenz, Medical Faculty, Department of Psychiatry and Psychotherapy, University Leipzig, Leipzig, Germany. E-mail: noah.lorenz@mementor.ch 
group effect remained stable over the period of 12 months. Further, sleep-related cognitions, safety behaviours, depression and somatization significantly decreased in the treatment group compared with the control group. On all sleep diary parameters, medium to large effects were revealed within the treatment group. Anxiety did not decrease significantly from pre- to postassessment. For all measures except somatization and anxiety significant within-group effects were found at 12-month follow-up assessment indicating long-lasting effects. Conclusions: This study adds evidence to the literature on unguided online interventions for insomnia, and indicates that online CBT-I can have substantial long-term effects on relevant sleep-related outcome parameters. Moreover, the results indicate that sleep-related cognitions and safety behaviour can be successfully altered with an unguided CBT-I intervention.

Keywords: insomnia, internet, randomized controlled trial, cognitive behavioural therapy, virtual coach, safety behaviours, cognition

\section{Introduction}

Insomnia has become a major public health concern, with far-reaching consequences for the health and well-being of affected people. In Western countries, sleep complaints are reported by one-third of the adult population, and 6-10\% meet the diagnostic criteria for insomnia disorder (Ohayon, 2011). Negative consequences of insomnia are diverse and include daytime fatigue, reduced daytime performance, mood impairments, physical complaints, and neuropsychological consequences such as difficulties with concentration and memory (Katz and McHorney, 2002; Léger et al., 2002). Moreover, evidence shows that insomnia often precipitates, exacerbates or prolongs a broad range of medical complaints such as mental or physical illnesses (Baglioni et al., 2010, 2011; Fernandez-Mendoza and Vgontzas, 2013; Pigeon et al., 2012; Roth and Roehrs, 2003; Spiegelhalder et al., 2012).

Cognitive behavioural therapy for insomnia (CBT-I) is an effective treatment with moderate to large effects on measures of sleep continuity and on subjective insomnia severity in general (Irwin et al., 2006; Morin et al., 2009; Trauer et al., 2015; Van Straten et al., 2017). Despite the fact that evidence-based treatments exist and despite the negative impacts of sleep disturbances, insomnia often remains unrecognized and untreated (Morin et al., 2006b). When insomnia symptoms are reported to professionals (usually to a primary care physician), treatment is normally limited to pharmacotherapy even though hypnotic medications can have severe side effects (Kripke et al., 2002).

In recent years, scalable and low-threshold internet-based self-help treatments have been developed for addressing a broad range of mental disorders and behavioural problems. Most internet-based treatments target disorder-specific perpetuating factors, such as dysfunctional behaviours, through CBT-based exercises and techniques. These techniques and exercises are either guided or unguided (Barak et al., 2008). Such internet-based self-help tools have also been developed and tested for insomnia. One meta-analysis found eleven published randomized controlled trials (RCTs) which had tested the effect of internet-delivered CBT-I (Zachariae et al., 2016). The global effect size (Hedge's $g$ ) was high, with 1.09 (CI 0.74-1.45) for insomnia severity and satisfactory for a broad range of secondary outcomes such as sleep onset latency, wake after sleep onset, or total sleep time. The meta-analysis included studies on guided and unguided interventions. The degree of personal clinical support was found to be associated with a larger effect size for sleep efficiency at post-treatment and a larger reduction on insomnia severity at follow-up assessment. However, some studies on unguided interventions reported 
high effect sizes as well. One web-based treatment called Sleepio (Espie et al., 2012) uses an animated avatar that provides all the content of the programme audio- and video-based with no clinical support needed. Sleepio was tested against a placebo and a treatment-as-usual group. High effect sizes on the primary outcome (sleep efficiency) were found when the treatment group was compared with both control groups.

Nevertheless, none of the 11 RCTs included in the meta-analysis reported long-term followup data. Only one recently published trial reported 12-month follow-up data of an online CBT-I intervention (indicating enduring effects; Ritterband et al., 2017). The intervention was a fully automated, interactive and tailored web-based treatment called SHUTi that included sleep restriction, stimulus control, cognitive restructuring, sleep hygiene and relapse prevention.

Although treatment effects seem substantial in online CBT-I, it has remained unclear if they are equivalent to traditional face-to-face approaches. Two recent studies compared online CBT-I against a CBT-I programme delivered face-to-face, and found conflicting results: Lancee et al. (2016) found that a face-to-face CBT-I treatment was superior to an online CBT-I intervention, whereas Blom et al. (2015) found that their online CBT-I intervention was noninferior, compared with a face-to-face treatment group.

Furthermore, it remains unclear which perpetuating factors that are hypothesized in theoretical models of insomnia are successfully targeted in online interventions. Conventional CBT-I, delivered face-to-face, mainly includes psychoeducation, sleep hygiene, stimulus control, sleep restriction, relaxation techniques and cognitive restructuring of unhelpful thoughts and beliefs about sleep (Morin, 2006). The effects of the behavioural components of CBT-I (i.e. stimulus control and sleep restriction) are well established (Morin et al., 2006a). Moreover, evidence shows that cognitions are also an important target for interventions (Harvey et al., 2007). Compared with healthy subjects, insomnia patients report more dysfunctional thoughts, attitudes and beliefs about sleep (Morin et al., 1993), and such dysfunctional cognitions play an important role in theoretical models of insomnia (Espie, 2002; Harvey, 2002; Lundh and Broman, 2000). Harvey (2002) suggests that attentional and perceptual processes, together with maladaptive beliefs and behaviour, contribute to the perpetuation of insomnia. According to this model, attentional processes include the monitoring of internal and external sleep-related threats, such as indicators of not getting enough sleep and of not functioning well during the day. Harvey (2002) postulates that these sleep-related threats lead to safety behaviours. Safety behaviours are maladaptive coping strategies (e.g. staying in bed longer than usual) that are executed to avoid the negative consequences of poor sleep. Harvey et al. (2007) suggest that such safety behaviours can be problematic for two reasons: first, safety behaviours may prevent patients from experiencing a non-confirmation of the feared negative consequence, and second, such behaviours may increase the probability that the anticipated negative consequences occur.

Cognitive and attentional biases as well as safety behaviours can be addressed and successfully altered in conventional, face-to-face CBT-I (e.g. Harvey et al., 2007). However, only a few studies have investigated whether cognitions and safety behaviours can be successfully altered in online interventions as well. Lancee et al. (2015) found that dysfunctional beliefs about sleep and safety behaviours were reduced after a guided online intervention for the treatment of insomnia. However, evidence on the successful delivery of cognitive techniques is scarce, particularly with respect to unguided internet-based treatments.

The aim of this study was to test the efficacy of an unguided internet-based treatment, 'mementor somnium', which involves behavioural and cognitive techniques for the treatment of 
insomnia. Aside from testing the general efficacy of the program, this study examines whether effects are stable over a long follow-up period and aims to determine whether cognitions and safety behaviour can be successfully altered using a virtual sleep coach.

\section{Method}

\section{Participants}

All participants were self-recruited via a study website. The link to the webpage was spread via e-mail, newspapers, clinics and clinical practitioners in Switzerland, Austria and Germany. People who expressed their interest in participation received an e-mail with study information and informed consent. Once written informed consent was given, participants received a link with screening questionnaires consisting of self-report measures and demographic questions. Inclusion screening criteria were: fluency in German, at least 18 years of age, having access to a computer and internet, and a minimum of 8 on the Insomnia Severity Index (ISI; Bastien et al., 2001). Exclusion criteria were working in shifts, ongoing psychological treatment, suicidality, psychotic disorder, alcohol abuse, a score higher than 19 on the Beck Depression Inventory revised (BDI-II; Beck et al., 1996) or suffering from a sleep disorder other than insomnia. Screening questionnaires were used for the following sleep disorders: obstructive sleep apnoea, restless legs syndrome and parasomnia. If the screening was positive, participants were excluded from the study. Medication was only an exclusion criterion if the dose was changed before or during the treatment or if signs of an abusive intake existed. Participants were asked if they took any medication. If that was the case, they were asked if they recently had changed the dose; in addition, screening questions concerning medication abuse were asked. If the screening was positive or if the dose had changed, people were excluded from the study. In the study information, participants were explicitly instructed not to change their regular medication or, otherwise, to report any changes in medication immediately to the study coordinator.

\section{Study design and procedure}

An RCT with two parallel arms was conducted: an online CBT-I group (treatment group), and a waiting list control group. After completion of the screening questionnaires, eligibility for inclusion was determined in a structured clinical interview by trained interviewers with at least a bachelor's degree in psychology. In the clinical interview, DSM-V criteria for insomnia disorder were checked. Participants who did not meet the criteria were excluded from the study. The interview took place via telephone. In total, 323 people showed interest in the study, 120 officially registered and completed the pre-study screening, and 56 participants met the inclusion criteria. The flowchart of the recruitment process, randomization, and assessments at pre-, post- and follow-up is presented in Fig. 1.

Participants were assigned to either the intervention or the waiting list condition in a 1:1 ratio using blocked randomization with 10 participants per block. Randomization was conducted by an independent person who had no contact with the participants. The interviewers were blinded to the randomization list. Sample size was calculated based on a power analysis performed with $\mathrm{G}^{*}$ Power (Faul et al., 2009). Based on other studies (Zachariae et al., 2016) a large effect 


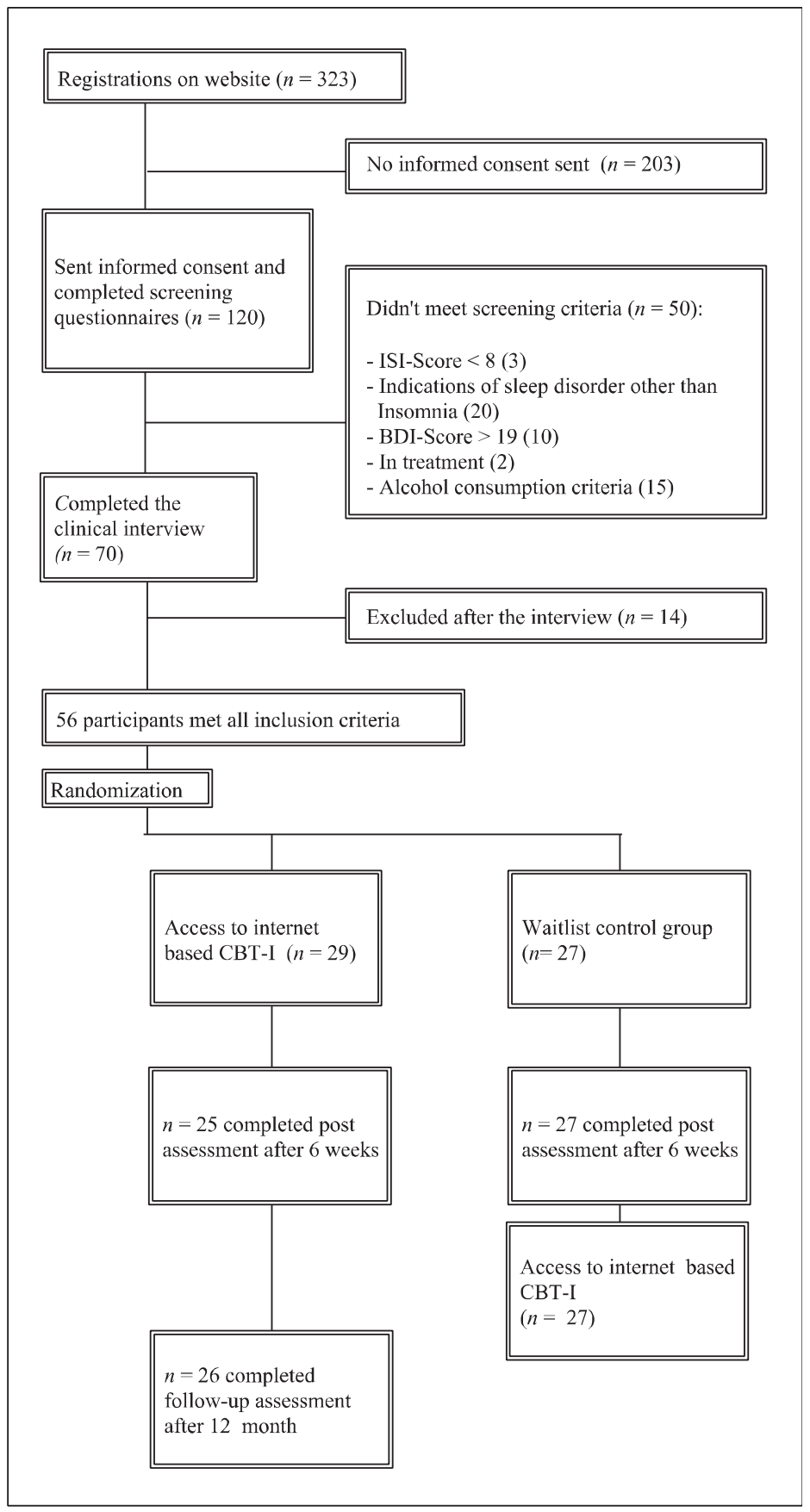

Figure 1. Flow of participants 
size of $f=0.5$ for treatment effects was expected; with a power of $80 \%$ and a significance level of $p<.01$ a minimal sample size of 40 participants (20 in each group) was calculated.

Duration of the intervention was 6 weeks. Information from screening was used as a baseline measure. Outcome measures at post-treatment and follow-up (12 months) were obtained electronically via a link to the online questionnaires sent to participants' e-mail addresses. Participants in the waitlist-condition received access to the treatment upon completion of the post-assessment. The study protocol was approved by the Ethics Committee Zurich. The trial was registered at Clinicaltrials.gov NCT02629913 and Swiss National Clinical Trials Portal.

\section{Measures}

The ISI (Bastien et al., 2001) was used as the primary outcome measure. This self-report questionnaire consists of seven items scored on a 5-point Likert scale, with higher scores indicating a higher degree of symptoms and perceived impact of sleep difficulties. Items are summed to a total score ranging from 0 to 28 . A total score of 0 to 7 is defined as normal, 8 to 14 as sub-threshold insomnia, 15 to 21 as moderate insomnia and above 21 as severe clinical insomnia (Bastien et al., 2001).

In the treatment group a sleep diary was used to measure process parameters. The sleep diary consisted of a morning and evening report. The morning report followed the guidelines of a sleep expert consensus on sleep diaries (Carney et al., 2012). The evening report was a slightly adapted version of the short evening report of the German Society of Sleep Medicine (Liendl and Hoffmann, 1999). Participants received e-mail reminders if they did not fill in the sleep diary. Moreover, sleep diary completion was automatically checked whenever the participant logged into the program, and participants could only continue the online treatment after adding missing reports for the prior three days. The following main parameters were calculated from the morning report of the sleep diary: sleep onset latency (SOL), wake after sleep onset (WASO), sleep efficiency (SE) and total sleep time (TST). In the evening report, participants rated 'overall sleep restfulness' (restfulness) and 'overall daytime performance' (daytime performance) on a scale from 0 to 100 .

The Anxiety and Preoccupation about Sleep Questionnaire (APSQ; Tang and Harvey, 2004) was used to measure sleep-related cognitions. This instrument consists of 10 statements, and participants rate their agreement with each statement on a 10-point scale. The Sleeprelated Behaviours Questionnaire (SRBQ; Ree and Harvey, 2004) was used to measure safety behaviour. The questionnaire consists of 32 items that assess insomnia-related safety behaviours. Items are rated on a 5-point Likert scale, adding to a sum score from 0 to 128 . Finally, to assess secondary symptoms, two well-established questionnaires were administered: the Beck Depression Inventory-II (BDI-II; Beck et al., 1996) for depression, and the Brief Symptom Inventory 18 (BSI-18; Derogatis, 2001), using only the subscales somatization (BSIS) and anxiety (BSI-A).

\section{CBT-I intervention}

The online intervention 'mementor somnium' consists of six fully automated sessions of CBT-I; every session contains different modules including psychoeducation, sleep restriction, relaxation, sleep hygiene, cognitive restructuring and changing sleep-related behaviours. A detailed description of the module content is provided in Table 1. The six treatment sessions 
Table 1. Content of 'mementor somnium'

\begin{tabular}{|c|c|c|}
\hline Module & Description & $\begin{array}{l}\text { Approximate } \\
\text { time needed }\end{array}$ \\
\hline Introduction & $\begin{array}{l}\text { General introduction, assessment of insomnia symptoms, } \\
\text { goal setting }\end{array}$ & $10 \mathrm{~min}$ \\
\hline $\begin{array}{l}\text { Sleep diary } \\
\text { introduction }\end{array}$ & $\begin{array}{l}\text { Introduction to the sleep diary, instructions on how to fill in } \\
\text { the morning and evening report }\end{array}$ & $10 \mathrm{~min}$ \\
\hline Sleep information & $\begin{array}{l}\text { Psychoeducation: basic information about sleep and } \\
\text { insomnia. The aim is to correct common misconceptions } \\
\text { about sleep which may contribute to the perpetuation of } \\
\text { insomnia }\end{array}$ & $20 \mathrm{~min}$ \\
\hline Practical exercise & $\begin{array}{l}\text { Learned knowledge is applied by taking on the role of a sleep } \\
\text { expert who must give advice to a patient suffering from } \\
\text { insomnia. Symptoms of the patient are similar to the } \\
\text { symptoms of the user }\end{array}$ & $15 \min$ \\
\hline $\begin{array}{l}\text { Insomnia vicious } \\
\text { cycle }\end{array}$ & $\begin{array}{l}\text { Introduction to an individual vicious cycle that can } \\
\text { contribute to the perpetuation of the insomnia disorder }\end{array}$ & $15 \mathrm{~min}$ \\
\hline Sleep schedule & $\begin{array}{l}\text { Introducing sleep restriction. The average total sleep time of } \\
\text { the last week is introduced as the new bedtime (sleep } \\
\text { window). Shortest sleep window is } 6 \text { hours. When the } \\
\text { average sleep efficiency of the past } 7 \text { days is }>85 \% \text {, the } \\
\text { sleep window can be extended by } 15 \text { min. The aim of this } \\
\text { module is to increase sleep pressure, which can improve } \\
\text { sleep efficiency }\end{array}$ & $15 \min$ \\
\hline Relaxation & $\begin{array}{l}\text { Introduction to the progressive muscle relaxation technique. } \\
\text { The aim is to decrease physical and mental tension in order } \\
\text { to enhance sleep onset latency and waking after sleep onset }\end{array}$ & $20 \mathrm{~min}$ \\
\hline Sleep behaviour & $\begin{array}{l}\text { Introduction to sleep hygiene rules and to the stimulus } \\
\text { control technique. The aim is to rebuild an association } \\
\text { between sleeping and bed by classical conditioning }\end{array}$ & $20 \mathrm{~min}$ \\
\hline Thoughts & $\begin{array}{l}\text { Cognitive restructuring. In an adaptive dialogue, different } \\
\text { typical thoughts that insomnia patients often have are } \\
\text { discussed. The aim is to identify dysfunctional } \\
\text { sleep-related thoughts and replace them with } \\
\text { sleep-compatible cognitions }\end{array}$ & $20 \min$ \\
\hline Daily decisions & $\begin{array}{l}\text { Typical sleep-related safety behaviour is presented in } \\
\text { vignettes. Different options of how to react to these } \\
\text { vignettes are given. The user has to decide on one option } \\
\text { which is later commented on by the animated sleep coach. } \\
\text { The aim is to demonstrate that reducing safety behaviour } \\
\text { can have positive effects on sleep }\end{array}$ & $15 \min$ \\
\hline Graduation & $\begin{array}{l}\text { The content of the different modules is briefly repeated and } \\
\text { tested in a quiz. The time after this intervention is planned }\end{array}$ & $15 \mathrm{~min}$ \\
\hline
\end{tabular}

were delivered chronologically, with a minimum waiting period of 48 hours between sessions. Participants were guided through the intervention by an animated sleep coach, which provided audio information supported by dynamic and interactive graphical content. At the beginning of the intervention, participants set goals on which they received continuous feedback based 
on the sleep diary data. A unique feature of the intervention is its tailored dialogue with the animated sleep coach. The coach asks questions throughout the treatment course, and delivers content based on the user's response behaviour.

\section{Statistical analyses}

Baseline group differences regarding demographic and clinical characteristics were analysed using independent $t$-tests (two-sided) and Pearson $\chi^{2}$-tests. To evaluate the effect of treatment on study outcome measures, linear mixed-effects analyses (using Restricted Maximum Likelihood as the estimator) were conducted. This statistical procedure is recommended for clinical trials with incomplete data (Beunckens et al., 2005). Treatment (mementor somnium versus wait-list control), time (pre- and post-assessment), and the interaction of Treatment $\times$ Time were entered as fixed factors, subject as a random factor, and ISI, APSQ, SRBQ, BDI-II, BSI-A and BSI-I as dependent variables in separate analyses. Effect sizes (Cohen's $d$ ) were calculated for changes within the treatment group from baseline to post- and follow-up assessment. Between-group effect sizes were calculated by using the mean pre/post-assessment change in the treatment group minus the mean pre/post-assessment change in the control group, divided by the pooled pre-assessment standard deviation as recommend by Morris (2008). Changes in sleep diary data were tested within the treatment group by using dependent $t$-tests (two-sided). For each of the six treatment weeks, daily sleep diary data were averaged to a weekly mean. As missing data could have influenced sleep diary and follow-up scores, multiple imputation was employed (Sterne et al., 2009). For the missing scores, ten separate datasets were generated. Pooled $t$-test results were reported. To evaluate whether any baseline variables were associated with treatment response, a logistic regression analysis was computed entering baseline variables as predictors and the ISI remission criterion as the dependent variable. Statistical analyses were conducted using IBM SPSS Statistics, version 23 and R.

\section{Results}

\section{Participant characteristics}

All demographic results for the different groups are reported in Table 2. For the analysis of group differences at baseline, independent sample $t$-tests were calculated. Significant differences were revealed for the ISI $(t(54)=2.25, p=.03)$ and BDI-II $(t(54)=2.23$, $p=.03)$, indicating higher scores on both variables for the treatment group. No significant differences were found on the remaining variables.

\section{Treatment attrition}

All participants of the control group and 25 participants of the treatment group completed the post-assessment (93\%). All participants that completed the post-assessment, plus one who had missed post-assessment, completed the 12-month follow-up assessment (94\% of all participants). The participant flow chart with detailed information is presented in Fig. 1.

On average, participants in the treatment group completed 5.62 of the six treatment sessions $(S D=0.74)$. All participants completed at least four treatment sessions. On average, the last 
Table 2. Demographic information

\begin{tabular}{|c|c|c|c|}
\hline Demographic information & $\begin{array}{l}\text { Treatment group } \\
(n=29)\end{array}$ & $\begin{array}{l}\text { Control group } \\
(n=27)\end{array}$ & Group comparison \\
\hline Age, M $(S D)$ & $41.72(17.31)$ & $44.04(20.05)$ & $t(54)=-0.46, p=.65$ \\
\hline Age range & $18-74$ & $19-77$ & \\
\hline $\begin{array}{l}\text { Sex, } n(\%) \\
\text { female }\end{array}$ & $21(72)$ & $18(67)$ & $\chi^{2}(1)=0.22, p=.64$ \\
\hline Country of birth, $n(\%)$ & & & $\chi^{2}(3)=6.15, p=.11$ \\
\hline Switzerland & $17(59)$ & $22(81)$ & \\
\hline Germany & $10(35)$ & $4(15)$ & \\
\hline Austria & $2(7)$ & $0(0)$ & \\
\hline Other & $0(0)$ & $1(4)$ & \\
\hline Education, $n(\%)$ & & & $\chi^{2}(5)=4.59, p=.47$ \\
\hline Obligatory school & $1(3)$ & $2(7)$ & \\
\hline Basic apprenticeship & $3(10)$ & $5(19)$ & \\
\hline Higher apprenticeship & $6(21)$ & $1(4)$ & \\
\hline College & $5(17)$ & $4(15)$ & \\
\hline University & $11(38)$ & $11(41)$ & \\
\hline Other & $3(10)$ & $4(15)$ & \\
\hline Professional situation, $n(\%)$ & & & $\chi^{2}(5)=1.00, p=.96$ \\
\hline Self-employed & $3(10)$ & $3(11)$ & \\
\hline Employee & $17(59)$ & $13(48)$ & \\
\hline Retired & $4(14)$ & $4(15)$ & \\
\hline Housewife/man & $1(3)$ & $2(7)$ & \\
\hline Unemployed & $2(7)$ & $3(11)$ & \\
\hline Other & $2(7)$ & $2(7)$ & \\
\hline Marital status, $n(\%)$ & & & $\chi^{2}(3)=2.34, p=.51$ \\
\hline Single & $11(38)$ & $14(52)$ & \\
\hline In cohabitation & $3(10)$ & $1(4)$ & \\
\hline Married & $12(41)$ & $11(41)$ & \\
\hline Divorced & $3(10)$ & $1(4)$ & \\
\hline Number of children, $\mathrm{M}(S D)$ & $0.79(1.35)$ & $0.74(1.10)$ & $t(54)=0.16, p=.87$ \\
\hline Housing situation, $n(\%)$ & & & $\chi^{2}(4)=4.47, p=.35$ \\
\hline Living alone & $4(14)$ & $6(22)$ & \\
\hline With parents & $1(3)$ & $4(15)$ & \\
\hline With partner & $19(66)$ & $11(41)$ & \\
\hline With children & $2(7)$ & $3(11)$ & \\
\hline Other & $3(10)$ & $3(11)$ & \\
\hline Prior in psychotherapy, $n(\%)$ & & & $\chi^{2}(1)=2.06, p=.15$ \\
\hline No & $20(69)$ & $23(85)$ & \\
\hline Yes & $9(31)$ & $4(15)$ & \\
\hline Takes medication, $n(\%)$ & & & $\chi^{2}(1)=0.57, p=.45$ \\
\hline No & $20(69)$ & $16(59)$ & \\
\hline Yes & $9(31)$ & $11(41)$ & \\
\hline
\end{tabular}


treatment session was completed after 29.76 days of treatment access $(S D=11.78)$. Participants completed the sleep diary an average of $85.90 \%$ of the days $(S D=18.33)$.

\section{Baseline characteristics}

The mean score of the ISI (Insomnia severity) for the total sample was $14.36(S D=3.66)$ at baseline, which corresponds to a clinical insomnia with medium severity (Bastien et al., 2001). For the other outcome measures the mean scores at baseline for the total sample were: BDIII (depression) mean $=8.27$ ( $S D=4.38)$, APSQ (sleep-related cognitions) mean $=60.16$ $(S D=19.14)$, SRBQ (sleep-related safety behaviour) mean $=41.25(S D=15.58)$, BSI-A (anxiety) mean $=3.57(S D=3.26)$ and BSI-S (somatization) mean $=2.21(S D=2.07)$. For the analysis of group differences at baseline, independent sample $t$-tests were calculated. Significant differences were revealed for the ISI $(t(54)=2.25, p=.03)$ and BDI-II $(t(54)=$ $2.23, p=.03)$ indicating higher scores on both variables for the treatment group. No significant differences were found on the remaining variables.

\section{Treatment effects}

Results of the statistical analyses are presented in Table 3. For the main outcome parameter, the ISI (Insomnia severity), a significant group $\times$ time interaction effect was found, indicating a marked improvement from pre-assessment to post-assessment in the treatment group and little change in the control group. The between-group effect size and the within-treatment-group effect size were large.

Apart from the statistically significant change on self-rated insomnia severity, results also indicated a clinically significant improvement on the remission criterion of an ISI score lower than 8 (Bastien et al., 2001). At post-treatment, 56\% of the participants in the treatment group had achieved remission, compared with $11 \%$ in the control group. A Pearson chi-squared test revealed a significant difference between the two groups on the remission criterion $\left(\chi^{2}(1, n=\right.$ $56)=11.89, p<.01)$. None of the baseline variables predicted whether participants achieved remission $\left(\chi^{2}(6, n=52)=5.485, p=.48\right)$.

For all sleep diary parameters, significant within-treatment differences between week 1 and week 6 were found, with effect sizes ranging from $d=0.45$ to $d=1.48$. SOL and WASO were reduced on average by 19 and $31 \mathrm{~min}$, respectively. TST increased an average of $35 \mathrm{~min}$. Statistical results of all pre-post differences (week 1 to week 5) are given in Table 4.

For the sleep-related cognitions and safety behaviours, significant group $\times$ time interaction effects emerged, indicating substantial improvements from pre- to post-assessment in the treatment group compared with the control group. In addition, there were significant group $\times$ time interaction effects on depression and somatization (BDI-II and BSI-S). In contrast to the other results, no significant group $\times$ time interaction effect on anxiety was revealed for the pre- to post-assessment comparison.

Large between-group and large within-treatment group effect sizes occurred for depression and sleep-related cognitions. For safety behaviour, somatization and anxiety small to medium between-group and small to medium within-treatment-group effect sizes occurred. For anxiety, 95\% confidence intervals of the within-treatment-group effect size included 0 indicating nonsignificance. 
Table 3. Mean, standard deviation and $n$ for each outcome measure divided by condition and assessment point, effect sizes and group $\times$ time interaction

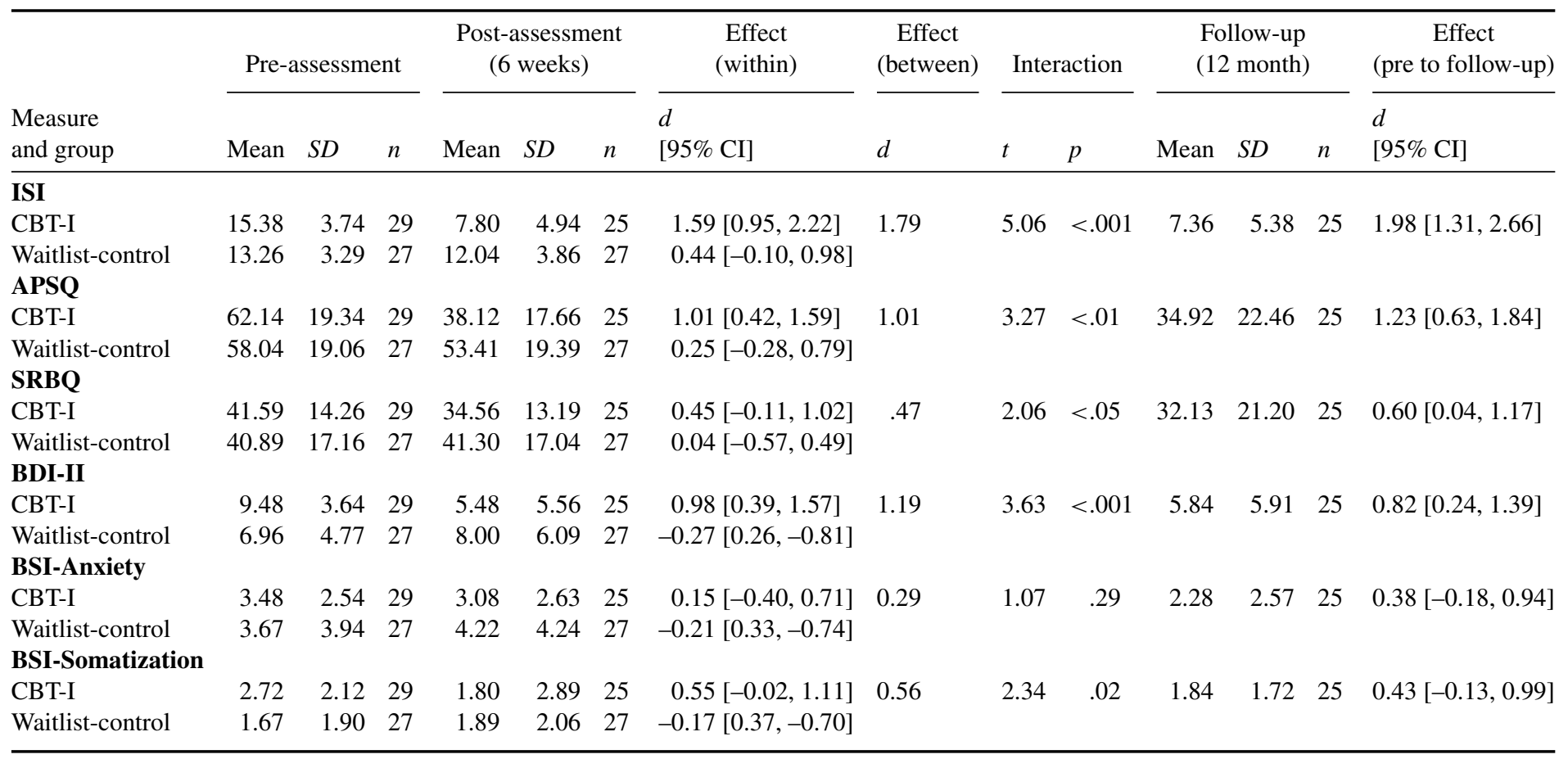

ISI, Insomnia Severity Index; APSQ, Anxiety and Preoccupation about Sleep Questionnaire; SRBQ, Sleep-related Behaviours Questionnaire; BDI-II, Beck Depression Inventory revised; BSI-Anxiety, Anxiety subscale of the Brief Symptom Inventory; BSI-Somatization, Somatization subscale of the Brief Symptom Inventory. 
Table 4. Comparison of week 1 and week 6 sleep diary parameters

\begin{tabular}{lccll}
\hline Parameter & Week 1 & Week 6 & Group & \\
& Mean $($ SEM $)$ & Mean $($ SEM) & comparison & Effect \\
\hline SE (\%) & $69.30(3.12)$ & $84.39(0.99)$ & $t(26)=-5.46, p<.01$ & $d=1.16$ \\
SOL (min) & $37.11(8.41)$ & $18.93(2.71)$ & $t(26)=2.38, p<.01$ & $d=0.50$ \\
WASO (min) & $53.78(7.04)$ & $22.32(3.07)$ & $t(26)=5.02, p<.01$ & $d=1.02$ \\
TST (h) & $348.19(17.94)$ & $383.60(7.65)$ & $t(26)=-2.29, p<.05$ & $d=0.45$ \\
Restfulness (\%) & $44.48(2.61)$ & $63.50(2.37)$ & $t(26)=-7.23, p<.01$ & $d=1.48$ \\
Daytime performance (\%) & $54.85(2.77)$ & $69.26(2.90)$ & $t(26)=-4.24, p<.01$ & $d=0.99$ \\
\hline
\end{tabular}

SE, sleep efficiency; SOL, sleep onset latency; WASO, wake after sleep onset; TST, total sleep time, $S E M$, standard error of the mean.

\section{Stability of treatment group effects}

A large within effect size between pre-assessment and follow-up regarding insomnia severity was found, indicating a long-lasting primary treatment effect. The same pattern occurred for sleep-related cognitions, safety behaviour and depression, with medium to large effect sizes. This indicates that secondary effects of the intervention were long-lasting as well. Statistical results of all pre- to follow-up differences are presented in Table 3.

\section{Discussion}

In this study, an unguided internet-based self-help intervention for insomnia (CBT-I) was compared with a waitlist control group. On the primary endpoint of self-reported insomnia severity, large between- and within-treatment group effect sizes and a significant interaction between the treatment and control group were observed, indicating a marked improvement on insomnia severity for the treatment group compared with the control group. The treatment effect was stable over a long follow-up period (12 months). This outcome appears comparable to therapist-delivered CBT-I (Riemann and Perlis, 2009) and to similar unguided CBT-I interventions (Espie et al., 2012; Ritterband et al., 2009). This study adds evidence to the literature on unguided internet interventions for insomnia and demonstrates that internet-based CBT-I can have substantial long-term effects on insomnia severity.

Interestingly, attrition rates were extremely low in this study (6-7\%) compared with other studies, in which rates were 14-48\% (Lancee et al., 2012, 2015). Thus, delivering CBT-I with a virtual sleep coach, including dynamic and interactive graphical content through an advanced web-based technology, might have a positive impact on the retention rate.

Taking a closer look on secondary outcome parameters, it was remarkable that the anxiety score of the BSI-18 did not change significantly from pre- to post-assessment, even though insomnia symptoms were already drastically reduced at post-assessment. There was a trend towards a significant effect at follow-up assessment, but the confidence interval still included 0 at the lower end ( -0.18 to 0.94$)$. Other studies had already revealed a close link between insomnia and anxiety disorder and highlighted a bidirectional association between these conditions (Jansson-Fröjmark and Lindblom, 2008). Therefore, it can be assumed that the non-significant effect could be due to the small sample size, reflecting a power problem. 
Furthermore, in line with previous studies, we found a substantial effect on depression symptoms. The symptom improvement measured with the BDI-II was significantly stronger in the intervention group compared with the control group. The same pattern occurred for the somatization score of the BSI. Remarkably, the treatment effect size on depression was still large at the 12-month follow-up assessment, indicating long-lasting transfer effects of internet-based CBT-I on depression.

Apart from testing the effectiveness of the internet-based self-help program, this study examined to what extent cognitions can be successfully altered with an unguided self-help intervention. The analyses showed a significant decrease of sleep-related worries and fears in the treatment group compared with the control group. These results indicate that the online intervention (mementor somnium) successfully modified maladaptive cognitions that have been shown to play an important role in perpetuating insomnia (Harvey, 2002). Furthermore, the intervention led to an adaptation of sleep-related safety behaviours in the treatment group, compared with the control group. Lancee et al. (2015) reported a medium within-group effect size $(d=.61)$ of their guided online intervention on sleep-related safety behaviour. This finding seems to be comparable to our own.

\section{Limitations}

Several limitations of the study must be mentioned. First, the small sample size did not allow for more sophisticated analysis for power reasons. With a bigger sample, it would have been possible to divide users into sub-samples and detect possible outcome mediators for them (e.g. improvements in safety behaviours or sleep-related cognitions). Second, the waitlist controlgroup did not have access to the sleep diary; this made it impossible to test for interaction effects on sleep diary parameters. Third, we did not include objective measurements (e.g. polysomnography) as outcome parameters due to the cost of such procedures. However, it should be noted that subjective measurements are standard in most RCTs for insomnia, and especially from the patient's point of view it is uncertain whether objective sleep parameters are more meaningful for measuring treatment effects of CBT-I (Buysse et al., 2006).

Despite these limitations, this study adds an important piece of evidence to current literature, showing that the burden of insomnia in the population can be addressed sustainably using fullyautomated CBT-I.

\section{Acknowledgements}

We thank all the participants who took part in the study. We acknowledge and thank mementor $\mathrm{GmbH}$ for making the online intervention available for this clinical trial.

Conflicts of interest: Noah Lorenz and Alexander Roetger are shareholders of mementor GmbH, the company that has developed the online intervention. Eva Birrer is part of the Advisory Board of mementor $\mathrm{GmbH}$.

Ethical statements: The study was approved by the ethical committee of Zurich, Switzerland: KEK-ZH-Nr. 2015-0279.

Financial support: No funds were raised for this research. The study was conducted by the Department of Psychology, University of Zurich, Switzerland. 


\section{Supplementary material}

To view supplementary material for this article, please visit https://doi.org/10.1017/ S1352465818000486

\section{References}

Baglioni, C., Battagliese, G., Feige, B., Spiegelhalder, K., Nissen, C., Voderholzer, U. et al. (2011). Insomnia as a predictor of depression: a meta-analytic evaluation of longitudinal epidemiological studies. Journal of Affective Disorders, 135, 10-19. http://doi.org/10.1016/j.jad.2011.01.011

Baglioni, C., Spiegelhalder, K., Lombardo, C. and Riemann, D. (2010). Sleep and emotions: a focus on insomnia. Sleep Medicine Reviews, 14, 227-238. http://doi.org/10.1016/j.smrv.2009.10.007

Barak, A., Hen, L., Boniel-Nissim, M. and Shapira, N. (2008). A comprehensive review and a metaanalysis of the effectiveness of internet-based psychotherapeutic interventions. Journal of Technology in Human Services, 26, 109-160. http://doi.org/10.1080/15228830802094429

Bastien, C., Vallières, A. and Morin, C. (2001). Validation of the Insomnia Severity Index as an outcome measure for insomnia research. Sleep Medicine, 2, 297-307. http://doi.org/doi:10.1016/ S1389-9457(00)00065-4

Beck, A., Steer, R. and Brown, G. (1996). Beck Depression Inventory-II. San Antonio, 12-15. http: //doi.org/10.1037/t00742-000

Beunckens, C., Molenberghs, G. and Kenward, M. G. (2005). Direct likelihood analysis versus simple forms of imputation for missing data in randomized clinical trials. Clinical Trials, 2, 379-386. http: //doi.org/10.1191/1740774505cn119oa

Blom, K., Tarkian Tillgren, H., Wiklund, T., Danlycke, E., Forssén, M., Söderström, A. et al. (2015). Internet- vs group-delivered cognitive behavior therapy for insomnia: a randomized controlled noninferiority trial. Behaviour Research and Therapy, 70, 47-55. http://doi.org/10.1016/j.brat.2015.05. 002

Buysse, D. J., Ancoli-Israel, S., Edinger, J. D., Lichstein, K. L. and Morin, C. M. (2006). Recommendations for a standard research assessment of insomnia. Sleep, 29, 1155-1173. http: //doi.org/10.1093/sleep/29.9.1155

Carney, C. E., Buysse, D. J., Ancoli-Israel, S., Edinger, J. D., Krystal, A. D., Lichstein, K. L. and Morin, C. M. (2012). The consensus sleep diary: standardizing prospective sleep self-monitoring. Sleep, 35, 287-302. http://doi.org/10.5665/sleep.1642

Derogatis, L. (2001). BSI 18, Brief Symptom Inventory 18: Administration, scoring and procedures manual. NCS Pearson, Incorporated.

Espie, C. A. (2002). Insomnia: conceptual issues in the development, persistence, and treatment of sleep disorder in adults. Annual Review of Psychology, 53, 215-243. http://doi.org/10.1146/annurev.psych. 53.100901 .135243

Espie, C. A., Kyle, S. D., Williams, C., Ong, J. C., Douglas, N. J., Hames, P. and Brown, J. S. L. (2012). A randomized, placebo-controlled trial of online cognitive behavioral therapy for chronic insomnia disorder delivered via an automated media-rich web application. Sleep, 35, 769-781. http: //doi.org/10.5665/sleep.1872

Faul, F., Erdfelder, E., Buchner, A. and Lang, A.-G. (2009). Statistical power analyses using G*Power 3.1: tests for correlation and regression analyses. Behavior Research Methods, 41, 1149-1160.

Fernandez-Mendoza, J. and Vgontzas, A. N. (2013). Insomnia and its impact on physical and mental health. Current Psychiatry Reports, 15, 418. http://doi.org/10.1007/s11920-013-0418-8

Harvey, A. G. (2002). A cognitive model of insomnia. Journal of Cognitive Psychotherapy, 18, 281-288. http://doi.org/10.1891/jcop.18.3.281.65649

Harvey, A. G., Sharpley, A. L., Ree, M. J., Stinson, K. and Clark, D. M. (2007). An open trial of cognitive therapy for chronic insomnia. Behaviour Research and Therapy, 45, 2491-2501. http://doi. org/10.1016/j.brat.2007.04.007 
Irwin, M. R., Cole, J. C. and Nicassio, P. M. (2006). Comparative meta-analysis of behavioral interventions for insomnia and their efficacy in middle-aged adults and in older adults 55+ years of age. Health Psychology, 25, 3-14. http://doi.org/10.1037/0278-6133.25.1.3

Jansson-Fröjmark, M. and Lindblom, K. (2008). A bidirectional relationship between anxiety and depression, and insomnia? A prospective study in the general population. Journal of Psychosomatic Research, 64, 443-449. http://doi.org/10.1016/j.jpsychores.2007.10.016

Katz, D. A. and McHorney, C. A. (2002). The relationship between insomnia and health-related quality of life in patients with chronic illness. Journal of Family Practice, 51, 229-235.

Kripke, D. F., Garfinkel, L., Wingard, D. L., Klauber, M. R. and Marler, M. R. (2002). Mortality associated with sleep duration and insomnia. Archives of General Psychiatry, 59, 131-136. http: //doi.org/10.1001/archpsyc.59.2.131

Lancee, J., Eisma, M. C., van Straten, A. and Kamphuis, J. H. (2015). Sleep-related safety behaviors and dysfunctional beliefs mediate the efficacy of online CBT for insomnia: a randomized controlled trial. Cognitive Behaviour Therapy, 44. http://doi.org/10.1080/16506073.2015.1026386

Lancee, J., van den Bout, J., van Straten, A. and Spoormaker, V. I. (2012). Internet-delivered or mailed self-help treatment for insomnia? A randomized waiting-list controlled trial. Behaviour Research and Therapy, 50, 22-29. http://doi.org/10.1016/j.brat.2011.09.012

Lancee, J., van Straten, A., Morina, N., Kaldo, V. and Kamphuis, J. H. (2016). Guided online or faceto-face cognitive behavioral treatment for insomnia: a randomized wait-list controlled trial. Sleep, 39, 183-191. http://doi.org/10.5665/sleep.5344

Léger, D., Guilleminault, C., Bader, G., Lévy, E. and Paillard, M. (2002). Medical and socio-professional impact of insomnia. Sleep, 25, 621-625. http://doi.org/10.1093/sleep/25.6. 621

Liendl, S. and Hoffmann, R. M. (1999). Compliance-probleme bei der bearbeitung von abend-morgenprotokollen - Entwicklung einer kurzversion der standardprotokolle der DGSM. Somnologie, 3, 73-77. http://doi.org/10.1007/s11818-999-0013-8

Lundh, L. G. and Broman, J. E. (2000). Insomnia as an interaction between sleep-interfering and sleep-interpreting processes. Journal of Psychosomatic Research, 49, 299-310. http://doi.org/10.1016/ S0022-3999(00)00150-1

Morin, C. M. (2006). Cognitive-behavioral therapy of insomnia. Sleep Medicine Clinics, 1, 375-386. http://doi.org/10.1016/j.jsmc.2006.06.008

Morin, C. M., Bootzin, R. R., Buysse, D. J., Edinger, J. D., Espie, C. A. and Lichstein, K. L. (2006a). Psychological and behavioral treatment of insomnia: update of the recent evidence (1998-2004). Sleep, 29, 1398-1414.

Morin, C. M., LeBlanc, M., Daley, M., Gregoire, J. P. and Merette, C. (2006b). Epidemiology of insomnia: prevalence, self-help treatments, consultations, and determinants of help-seeking behaviors. Sleep Medicine, 7, 123-130. http://doi.org/10.1016/j.sleep.2005.08.008

Morin, C. M., Stone, J., Trinkle, D., Mercer, J. and Remsberg, S. (1993). Dysfunctional beliefs and attitudes about sleep among older adults with and without insomnia complaints. Psychology and Aging, 8, 463-467. http://doi.org/10.1037/0882-7974.8.3.463

Morin, C. M., Vallieres, A., Guay, B., Ivers, H., Savard, J., Merette, C. et al. (2009). Cognitive behavioral therapy alone and with medication for persistent insomnia [2]. Journal of the American Medical Association, 301, 2005-1015. http://doi.org/10.1001/jama.2009.1282

Morris, S. B. (2008). Estimating effect sizes from pretest-posttest-control group designs. Organizational Research Methods, 11, 364-386. http://doi.org/10.1177/1094428106291059

Ohayon, M. M. (2011). Epidemiological overview of sleep disorders in the general population. Sleep Medicine Research, 2, 1-9. http://doi.org/s

Pigeon, W. R., Pinquart, M. and Conner, K. (2012). Meta-analysis of sleep disturbance and suicidal thoughts and behaviors. Journal of Clinical Psychiatry, 73, e1160-e1167. http://doi.org/10.4088/JCP. $11 \mathrm{r} 07586$ 
Ree, M. J. and Harvey, A. G. (2004). Investigating safety behaviours in insomnia: the development of the Sleep-related Behaviours Questionnaire (SRBQ). Behaviour Change, 21, 26-36. http://doi.org/ 10.1375/bech.21.1.26.35971

Riemann, D. and Perlis, M. L. (2009). The treatments of chronic insomnia: a review of benzodiazepine receptor agonists and psychological and behavioral therapies. Sleep Medicine Reviews. http://doi.org/ 10.1016/j.smrv.2008.06.001

Ritterband, L. M., Thorndike, F. P., Gonder-Frederick, L. A., Magee, J. C., Bailey, E. T., Saylor, D. K. and Morin, C. M. (2009). Efficacy of an internet-based behavioral intervention for adults with insomnia. Archives of General Psychiatry, 66, 692-698. http://doi.org/10.1016/S8756-3452(09) 79335-1

Ritterband, L. M., Thorndike, F. P., Ingersoll, K. S., Lord, H. R., Gonder-Frederick, L., Frederick, C. et al. (2017). Effect of a web-based cognitive behavior therapy for insomnia intervention with 1-year follow-up. JAMA Psychiatry, 74, 68. http://doi.org/10.1001/jamapsychiatry.2016.3249

Roth, T. and Roehrs, T. (2003). Insomnia: epidemiology, characteristics, and consequences. Clinical Cornerstone, 5, 5-15. http://doi.org/10.1016/S1098-3597(03)90031-7

Spiegelhalder, K., Regen, W., Feige, B., Holz, J., Piosczyk, H., Baglioni, C. et al. (2012). Increased EEG sigma and beta power during NREM sleep in primary insomnia. Biological Psychology, 91, 329-333. http://doi.org/10.1016/j.biopsycho.2012.08.009

Sterne, J. A. C., White, I. R., Carlin, J. B., Spratt, M., Royston, P., Kenward, M. G. et al. (2009). Multiple imputation for missing data in epidemiological and clinical research: potential and pitfalls. BMJ, 338, b2393-b2393. http://doi.org/10.1136/bmj.b2393

Tang, N. and Harvey, A. (2004). Correcting distorted perception of sleep in insomnia: a novel behavioural experiment? Behaviour Research and Therapy, 42, 27-39. http://doi.org/10.1016/S0005-7967(03) 00068-8

Trauer, J. M., Qian, M. Y., Doyle, J. S., Rajaratnam, S. M. W. and Cunnington, D. (2015). Cognitive behavioral therapy for chronic insomnia: a systematic review and meta-analysis. Annals of Internal Medicine, 163, 191-204. http://doi.org/10.7326/M14-2841

Van Straten, A., Van Der Zweerde, T., Kleiboer, A., Cuijpers, P., Morin, C. M. and Lancee, J. (2017). Cognitive and behavioral therapies in the treatment of insomnia: a meta-analysis. Sleep Medicine Reviews. http://doi.org/10.1016/j.smrv.2017.02.001

Zachariae, R., Lyby, M. S., Ritterband, L. M. and O'Toole, M. S. (2016). Efficacy of internetdelivered cognitive-behavioral therapy for insomnia - a systematic review and meta-analysis of randomized controlled trials. Sleep Medicine Reviews, 30, 1-10. http://doi.org/10.1016/j.smrv.2015. 10.004 